\title{
Wolf-Rayet and LBV Nebulae as the Result of Variable and Non-spherical Stellar Winds
}

\author{
Mordecai-Mark Mac Low \\ Max-Planck-Institut für Astronomie, Königstuhl 17, D-69117 Heidelberg, Germany
}

\begin{abstract}
The physical basis for interpreting observations of nebular morphology around massive stars in terms of the evolution of the central stars is reviewed, and examples are discussed, including NGC 6888, OMC-1, and $\eta$ Carinae.
\end{abstract}

\section{Introduction}

The nebulae observed around massive, post-main sequence stars appear to consist of material ejected by the central stars during earlier phases of their evolution, rather than ambient interstellar matter. Models of these nebulae can be used to constrain the mass-loss history of the stars, giving an important input for stellar evolution models. Understanding the structure of these nebulae also clarifies the initial conditions for the resulting supernova remnants, which will interact with the circumstellar material for most of their observable lifetimes before encountering the surrounding interstellar medium.

A strong stellar wind sweeps the surrounding interstellar gas into a stellar wind bubble as shown in Figure 1. The stellar wind expands freely until it reaches a termination shock. If this shock is adiabatic, the hot gas sweeps up the surrounding ISM into a dense shell, forming a pressure-driven or energyconserving bubble that sweeps up the surrounding ISM into a dense shell growing as $R \propto t^{3 / 5}$ in a uniform medium (Castor, McCray, \& Weaver 1975). Should the termination shock be strongly radiative due to high densities or low velocities in the wind, the bubble only conserves momentum and will grow as $R \propto t^{1 / 2}$ (Steigman, Strittmatter, \& Williams 1975). For more general discussions of blast waves in non-uniform media, see Ostriker \& McKee (1988), and Bisnovatyi-Kogan \& Silich (1995), as well as Koo \& McKee (1990).

When these stars leave the main sequence, they pass through phases of greatly increased mass loss. These slow, dense winds expand into the rarefied interior of the main sequence bubble until their ram pressure $\rho_{w} v_{w}^{2}$ drops below the pressure of the bubble. (Should the main sequence bubble have cooled relatively quickly, this may never occur.) As the mass loss rate and velocity of the central wind vary during the post-main sequence evolution of the central star, these denser winds can in turn be swept up, producing the observed ring nebulae around evolved massive stars.

During their evolution, these nebulae are subject to a number of hydrodynamical instabilities, as well as thermal instabilities (e. g. Strickland \& 


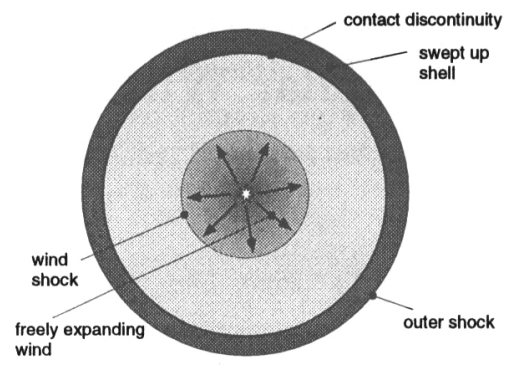

Fig. 1. Stellar wind bubble structure.

Blondin 1995). I explain how understanding the physical basis of the hydrodynamical instabilities gives insight into the dynamics of observed nebulae. High-resolution observations of nebular morphology can thus be used to constrain the mass-loss history of the central star.

I then describe how typical stellar evolutionary scenarioes can generate observed nebular morphologies, and show semi-analytic and numerical models derived from these scenarioes. For example, a star with a stellar wind varying from fast to slow and back again will have a clumpy circumstellar nebula due to hydrodynamical instabilities in the shell (Garcia-Segura, Langer \& Mac Low 1996). Nonspherical winds and stellar motion can add to the morphological richness of the resulting nebulae, as in the nebula around $\eta$ Car (Langer, García-Segura, \& Mac Low 1998). A recent review of this topic is Frank (1998).

\section{Shell Instabilities}

Gas swept up by a stellar wind will usually be subject to different instabilities. An adiabatic, decelerating shell with a density contrast across the shock of less than 10 in a uniform medium is stable. However, relaxing any of these constraints will lead to instabilities as I now describe.

\subsection{Rayleigh-Taylor Instability}

If the swept-up shell is denser than the stellar wind, as will be true in virtually all cases where a shell exists at all, the shell will be subject to RT instabilities if the contact discontinuity between the shocked stellar wind and the shell accelerates. This can be due to an external density gradient steeper than $r^{-2}$ or to a sufficiently fast increase in the power of the central stellar wind, though these two mechanisms will lead to different shell morphologies, as I discuss below.

The RT instability occurs when the effective gravity due to acceleration points from a denser to a more rarefied gas. We can understand its driving 


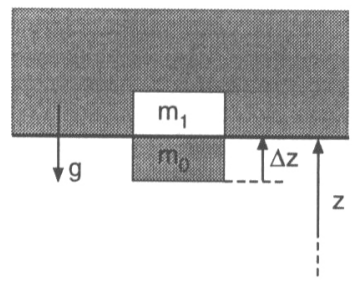

Fig. 2. Rayleigh-Taylor instability

mechanism by considering how the potential energy will change if we interchange a parcel of dense gas having mass $m_{1}$ with a parcel of more rarefied gas having $m_{0}<m_{1}$, as shown in Figure 2 . The potential energy before the interchange is given by $E_{i}=m_{1} g z+m_{0} g(z-\Delta z)$ which is greater than the potential energy after the interchange $E_{f}=m_{0} g z+m_{1} g(z-\Delta z)$ due to the difference in the masses. The decrease in potential energy drives an exponentially growing interchange of the two fluids.

When a RT instability occurs due to an external density gradient, dense fragments of shell are left behind as the less dense interior expands out beyond them, creating the characteristic bubble and spike morphology seen, for example, in models of superbubble blowout (e. g. Mac Low, McCray, \& Norman 1989). The Wolf-Rayet ring nebula NGC 6888 shown in Figure 3 provides another example. Here a fast, rarefied Wolf-Rayet wind has swept up the slow, dense red supergiant wind that preceded it. While it was still sweeping up the slow wind, it was marginally stable to RT instabilities. However, at the outer edge of the slow wind, the sharp density gradient triggers RT instabilities, as modelled by García-Segura \& Mac Low (1995) with the astrophysical gas dynamics and magnetohydrodynamics code ZEUS $^{1}$ (Stone \& Norman 1992).

On the other hand, when a RT instability occurs due to an increase in power of the driving wind, some of the dense fragments of shell actually get shot out ahead of the bulk of the fragmenting shell, producing a markedly different morphology (Stone, Xu, \& Mundy 1995). Although these fragments represent only a small fraction of the total mass of the shell, they can produce a very striking set of bow shocks in their wake. An example of this occurring around one or more pre-main sequence stars is given by the "bullets" observed around OMC-1 (Lane 1989, Allen \& Burton 1993) in the Orion star forming region, as confirmed by McCaughrean \& Mac Low (1997).

\subsection{Vishniac Instability}

If a pressure-driven shell is decelerating, but thin, with a density contrast across the shock of at least 25 for a stellar-wind bubble expanding into a


at lca@ncsa.uiuc.edu
} 

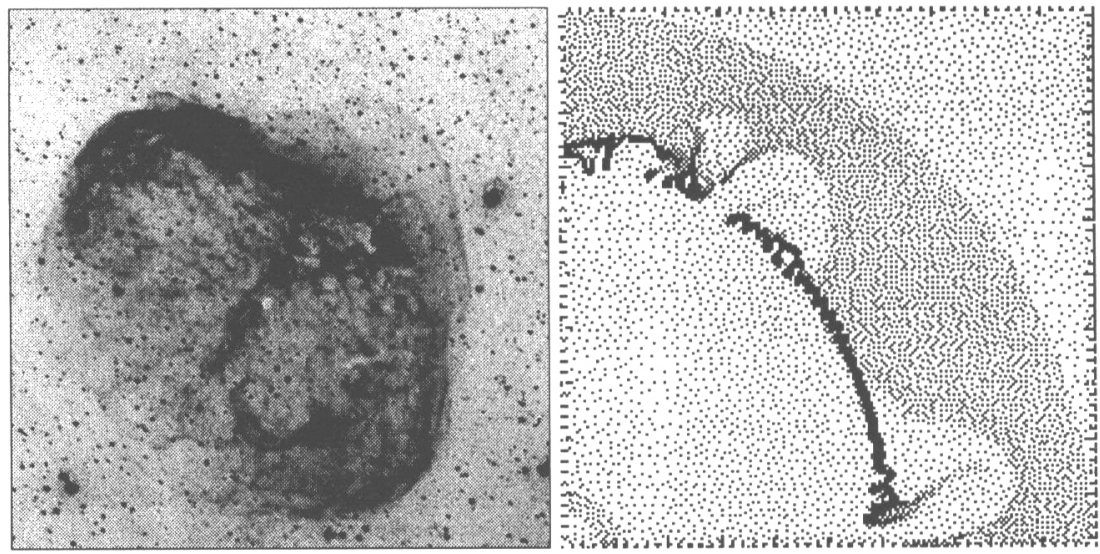

Fig. 3. Comparison between morphology of NGC 6888 in O [III] (in an image taken by K. B. Kwitter with the Burrell-Schmidt telescope of the Warner and Swasey Observatory, Case Western Reserve University), and a numerical simulation of RT instability due to a fast wind sweeping over the end of a slow, dense wind (García-Segura \& Mac Low 1995). The model image shows a cross-section of the density structure in grayscale with black indicating high density and white low density.

uniform medium (Ryu \& Vishniac 1988), or 10 for a point explosion (Ryu \& Vishniac 1987), it will be subject to the Vishniac overstability (Vishniac 1983). This has been confirmed experimentally using blast waves generated by high-powered lasers propagating into gases with low and high adiabatic indexes (Grun et al. 1991).

The mechanism of the Vishniac overstability can be understood by considering a thin, decelerating shell driven from within by a high-pressure region, as shown in Figure 4. From within it is confined by thermal pressure acting normal to the shell surface, as adjacent regions can communicate with each other by sound waves, while from outside it is confined by ram pressure acting parallel to the velocity of propagation, as the shell moves supersonically into the surrounding gas. In equilibrium, these two forces remain in balance. Should the shell be perturbed, however, the thermal pressure will continue to act normally, but the ram pressure will now act obliquely, giving a transverse resultant force that drives material from "peaks" into "valleys" of the shell. The denser valleys will be decelerated less than the rarefied peaks, however, so that the positions of peaks and valleys are interchanged after some time. Vishniac (1983) showed that this overstable oscillation can grow as fast as $t^{1 / 2}$. It saturates when the transverse flows in the shell become supersonic and form transverse shocks, so that the end result of Vishniac instability is a shell with transonic turbulence and moderate perturbations (Mac Low \& Norman 1993). 


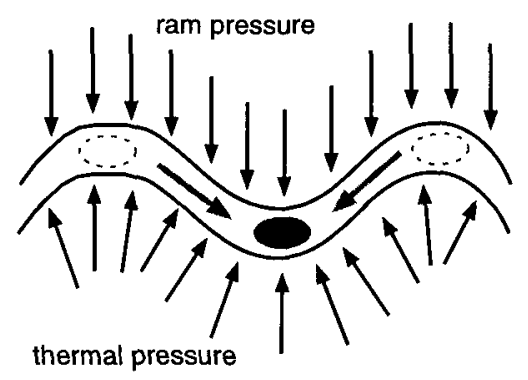

Fig. 4. Vishniac instability mechanism

\subsection{Nonlinear Thin Shell Instability}

Should the driving wind cool immediately behind its termination shock, for example because of exceptionally high mass-loss rates, it can form a decelerating shell that is momentum-driven rather than pressure-driven, so that it is effectively confined on both sides by ram pressure from shocks. Such a shell is not subject to the Vishniac instability, and is, in fact, linearly stable. However Vishniac (1994) has shown that if the shell is strongly perturbed, it will still be subject to a nonlinear thin shell instability (NTSI). When the shocks are oblique enough to the direction of flow, they will bend the streamlines passing through them, so that mass is driven towards the extrema of the perturbation. Numerical simulations by Blondin \& Marks (1996), using a piecewise parabolic hydrocode called VH-1, have shown that the end result is a catastrophic breakup of the shell into a turbulent layer that grows in time.

\section{A Final Example: Eta Carinae}

As an example of how knowledge of these different instabilities can be used to constrain the evolution of a star, consider the example of the Homunculus Nebula around $\eta$ Car. Langer, García-Segura, \& Mac Low (1998) computed several two-dimensional models of it using ZEUS, following a basic scenario in which a luminous blue star with a fast stellar wind undergoes an outburst during which it has a much slower and denser wind strongly shaped by rotation, as described by Bjorkman \& Cassinelli (1993), but then reverts to its previous state with a fast, rarefied wind. They chose two different values for the post-outburst wind, one consistent with current observed values of $\dot{M}=1.3 \times 10^{-3} M_{\odot} \mathrm{yr}^{-1}$ and $v_{w}=1300 \mathrm{~km} \mathrm{~s}^{-1}$, and one with a faster, lower mass loss wind having $\dot{M}=1.7 \times 10^{-4} M_{\odot} \mathrm{yr}^{-1}$ and $v_{w}=1800 \mathrm{~km} \mathrm{~s}^{-1}$. As shown in Figure 5, the slower, denser wind cools upon shocking, forming a momentum-driven shell that fragments due to the NTSI, producing a sharp, spiky shell morphology. On the other hand, the faster wind does not cool completely, and forms a bubble subject to Vishniac instabilities, giving it 


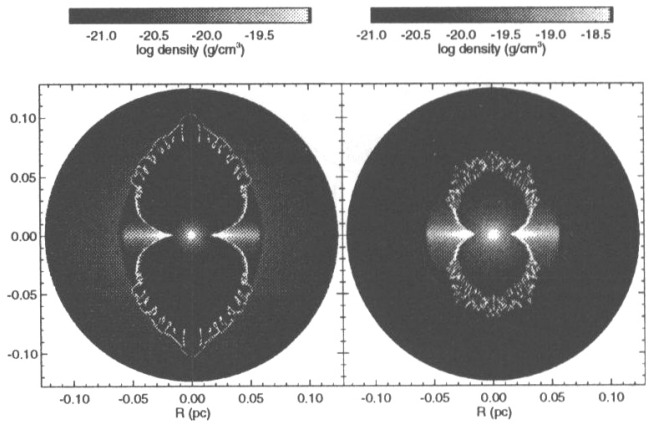

Fig. 5. Two-dimensional density distributions from models of $\eta$ Car by Langer, Garcia-Segura, \& Mac Low (1998) with faster and slower post-outburst wind showing Vishniac instabilities and the NTSI respectively. Note how the faster wind model resembles a cross-section through the cauliflower-like observed lobes.

a much more curved, cauliflower-like appearance. Comparison to the highresolution observations (Humphreys \& Davidson 1994; Morse, Davidson, \& Ebbets 1997) reveals that the actual morphology strongly resembles a threedimensional version of the model with the faster wind. Langer et al. (1998) suggest that this reflects the typical behavior of the wind over the century since the outburst, and that the current wind properties are actually exceptional, and perhaps even indicative of another outburst on its way. This suggestion is supported by the gradual brightening of $\eta$ Car over the last decades (Humphreys \& Davidson 1994).

This work has made use of the NASA Astrophysical Data System Abstract Service. I thank the organizers for their invitation and their support of my attendence at this meeting.

\section{References}

Allen, D. A., \& Burton, M. G. (1993): Explosive ejection of matter associated with star formation in the Orion nebula. Nature, 363, 54-56

Bjorkman, J., \& Cassinelli, J. (1993): Equatorial disk formation around rotating stars due to ram pressure confinement by the stellar wind. Astrophys. J., 409, $429-449$

Bisnovatyi-Kogan, G. S., \& Silich, S. A. (1995): Shock-wave propagation in the nonuniform interstellar medium. Rev. Mod. Phys., 67, 661-712

Blondin, J. M., \& Marks, B. S. (1996): Evolution of cold shock-bounded slabs. New Astron., 1, 235-244

Castor, J., McCray, R., \& Weaver, R. (1975): Interstellar Bubbles. Astrophys. J. (Letters), 200, L107-L110 
Frank, A. (1998): Bipolar Outflows and the Evolution of Stars. New Astron. Rev., in press (astro-ph/9805275)

García-Segura, G., Langer, N., \& Mac Low, M.-M. (1996): The hydrodynamic evolution of circumstellar gas around massive stars. II. The impact of the time sequence O star $\rightarrow$ RSG $\rightarrow$ WR star. Astron. Astrophys., 316, 133-146

García-Segura, G., \& Mac Low, M.-M. (1995): Wolf-Rayet Bubbles. II. Gasdynamical Simulations. Astrophys. J., 455, 160-174

Grun, J., Stamper, J., Manka, C., Resnick, J., Burris, R., Crawford, J., \& Ripin, B. H. (1991): Instability of Taylor-Sedov blast waves propagating through a uniform gas. Phys. Rev. Lett., 66, 2738-2741

Humphreys, R. M., Davidson, K. (1994): The luminous blue variables: Astrophysical geysers. Publ. Astron. Soc. Pacific, 106, 1025-1051

Koo, B.-C., \& McKee, C. F. (1990): Dynamics of adiabatic blast waves in media of finite mass. Astrophys. J., 354, 513-528

Lane, A. P. (1989): Near Infrared Imaging of H2 Emission from Herbig-Haro Objects and Bipolar Flows. Proceedings of the ESO Workshop on Low Mass Star Formation and Pre-main Sequence Objects (ESO, Garching bei München), 331

Langer, N., García-Segura, G., \& Mac Low, M.-M. (1998): "Giant Outbursts of Luminous Blue Variables and the Formation of the Homunculus Nebula Around $\eta$ Carinae. Astrophys. J. (Letters), submitted

Mac Low, M.-M., \& McCaughrean, M. J. (1997): The OMC-1 Molecular Hydrogen Outflow as a Fragmented Stellar Wind Bubble. Astron. J., 113, 391-400

Mac Low, M.-M., McCray, R., \& Norman, M. L. (1989): Superbubble blowout dynamics. Astrophys. J., 337, 141-154

Mac Low, M.-M., \& Norman, M. L. (1993): Nonlinear growth of dynamical overstabilities in blast waves. Astrophys. J., 407, 207-218

Morse, J., Davidson, K., Ebbets, D. (1997): Multi-band WFPC2 Imaging of Eta Carinae. Bull. Amer. Astron. Soc., 29

Ostriker, J. P., \& McKee, C. F. (1988): Astrophysical blastwaves. Rev. Mod. Phys., 60, 1-68

Ryu, D., \& Vishniac, E. T. (1987): The growth of linear perturbations of adiabatic shock waves. Astrophys. J., 313, 820-841

Ryu, D., \& Vishniac, E. T. (1988): A linear stability analysis for wind-driven bubbles. Astrophys. J., 331, 350-358

Steigman, G., Strittmatter, P. A., Williams, R. E. (1975): The Copernicus observations--Interstellar or circumstellar material. Astrophys. J., 198, 575-582

Stone, J. M., \& Norman, M. L. (1992): ZEUS-2D: A radiation magnetohydrodynamics code for astrophysical flows in two space dimensions. I. The hydrodynamic algorithms and tests. Astrophys. J. Suppl., 80, 753-790

Stone, J. M., Xu, J., \& Mundy, L. G. (1995): Formation of Bullets by Hydrodynamical Instabilities in Stellar Outflow. Nature, 377, 315-316.

Strickland, R., \& Blondin, J. M. (1995): Numerical Analysis of the Dynamic Stability of Radiative Shocks. Astrophys. J., 449, 727-738

Vishniac, E. T. (1983): The dynamic and gravitational instabilities of spherical shocks. Astrophys. J., 274, 152-167

Vishniac, E. T. (1994): Nonlinear instabilities in shock-bounded slabs. Astrophys. $J ., 428,186-208$ 


\section{Discussion}

A. Feldmeier: Do you see interactions between the Vishniac instability and the Rayleigh-Taylor instability in your thin-shell simulations?

M. MacLow: Yes. For example, in García-Segura \& MacLow (1995) we modelled the behaviour of a Vishniac-unstable shell as it ran off the edge of a red supergiant wind and became Rayleigh-Taylor unstable. The Vishniac instability tends to act as a seed for R-T, by determining at least the initial wavelengths on which it acts.

L. Kaper: Could you comment on the time scales for growth of the different instabilities? Would it be possible that when the nonlinear, thin-shell instability operates the materialinvolved becomes so fragmented that it becomes difficult to observe?

M. MacLow: The Rayleigh-Taylor instability has an exponential growth rate, while both the Vishniac instability and the nonlinear, thin-shell instability have power-law growth rates. Time constants depend on many things, but will usually be somewhat shorter than the dynamical time. As far as observability goes, fragmentation per se will not reduce observability, except insofar as it creates a thicker, less dense shell. On the other hand, if the fragments are denser, their emission measure might actually increase.

H. Lamers: There is evidence based on a comparison between the nebular abundances and the abundances of the central object of $\eta$ Car (obtained from high-resolution HST/UV spectra) that the star we see now (the LBV) is not the star that exploded. So one cannot derive the post-ejection wind properties from observations of the central object (Lamers et al., ApJ Letters, August 1998).

M. MacLow: This will naturally change the interpretation of our results, but the constraint of a rarified, fast wind having been dynamically important for the last century remains.

S. Shore: What happens in a full 3D model of the Vishniac instability? Would you expect enhanced filamentation when vorticity is not a constraint? M. MacLow: This is an interesting question that is waiting for someone to put the time in to answer. We have the technology available to do these models now. I expect some enhanced filamentation as seen in the Grun et al. experiment. However, they also observed saturation suggesting that saturation by transverse shocks as described by MacLow \& Norman (1992) will still occur.

G. Mellema: Can you explain why the polar lobes in your $\eta$ Car models appear to be more unstable than the equatorial regions?

M. MacLow: Numerical dissipation is probably suppressing some KelvinHelmholtz instabilities. Secondly, other instabilities are not appearing strongly 
because the shell is decelerating much more strongly at the equator due to the blowout of the polar lobes.

A. Moffat: All your calculations of wind interactions assume initially smooth winds. Then follows the obvious question as to the impact of clumped winds for which growing evidence suggests high degrees of multi-scale clumping. Even if you have not done any such calculations, can you guess what might happen?

M. MacLow: Yes, all of our models assume smooth winds. I am by no means satisfied with this assumption, so much so that I have devoted most of my time for the past year or so to modelling hydrodynamic and MHD turbulence with the ultimate intention of blowing winds into it to understand the behaviour of blast winds in real turbulent media. Simple models of dense clumps in a uniform density background will not be enough, that is already clear. The effects should include enhanced instabilities and thicker shells, perhaps also faster expansion.

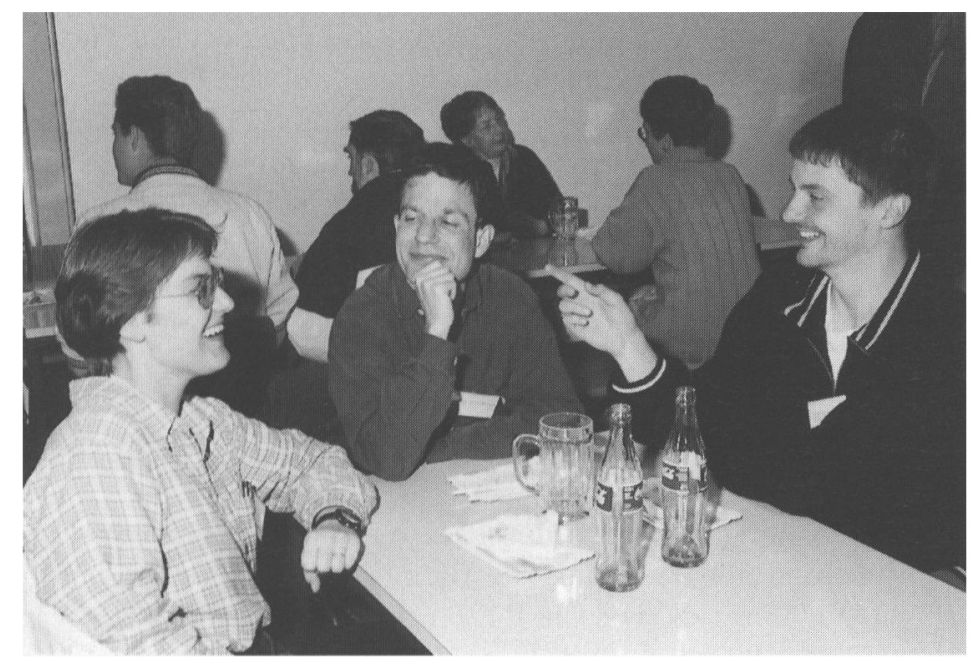

Kerstin Weis, Mordecai-Mark MacLow and Lars Koesterke 